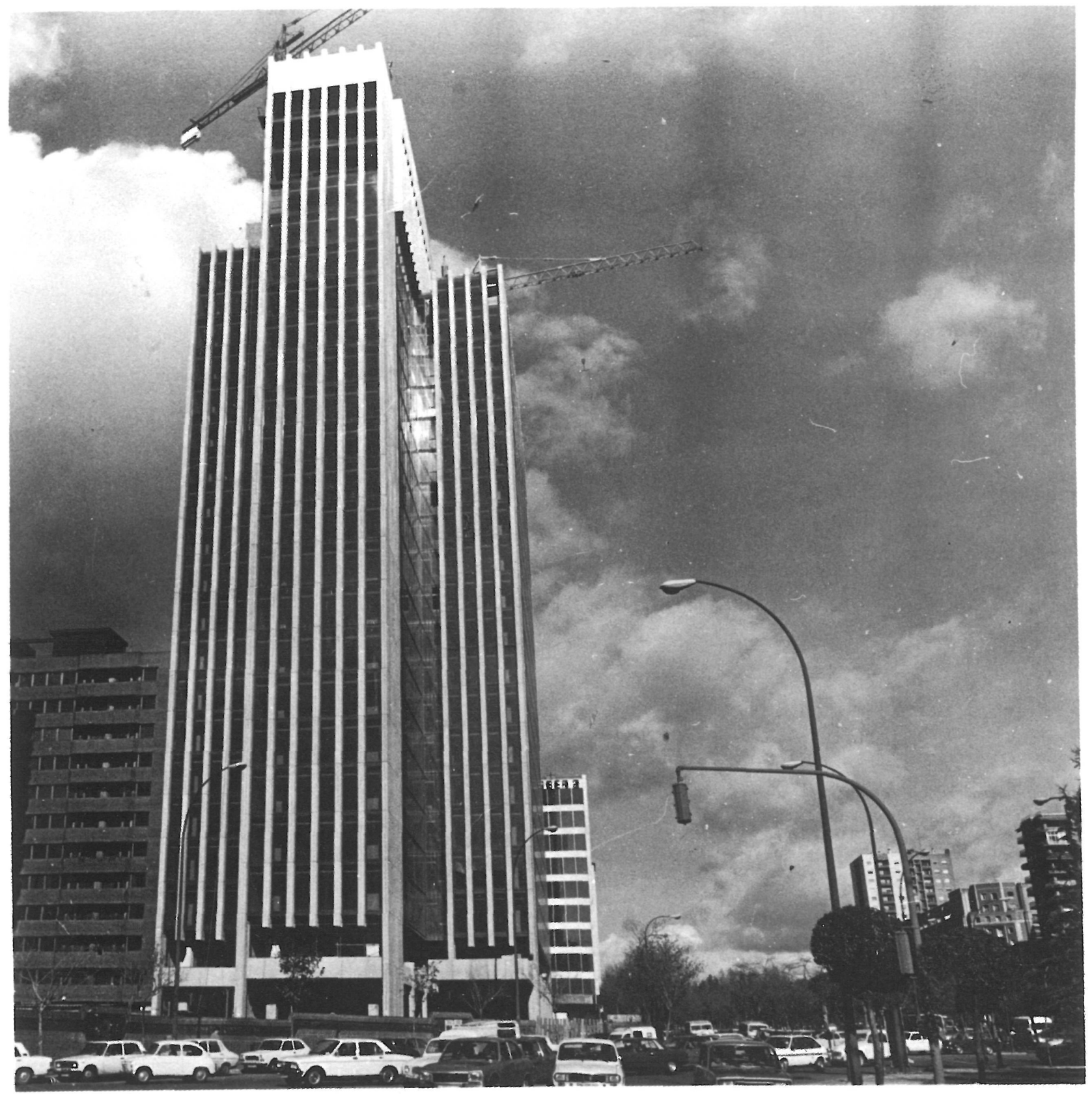

\title{
Características del hormigón para prefabricados de fachada
}

\author{
Antonio Puerta \\ SCHOKBETON ESPAÑOLA, S. A.
}

$884-2$

\section{sinopsis}

Se estudian en este articulo las ventajas que ofrecen, ol hormigón chocado en la actualidad y el hormigón polimerizado en el futuro, sobre los materiales tradicionalmente empleados en la prefabricación de fachadas: piedras y mármoles naturales de poca porosidad, piedra artificial y hormigón vibrado.

Además de describirse las características de estos materiales, se dan unas indicaciones para la perfecta ejecución de las obras y ciertas recomendaciones al objeto de que la solución adoptada sea la más estética, racional, permanente y económica posible. 


\section{EL HORMIGON ARQUITECTONICO}

Existe cierta confusión sobre el material que debe emplearse en los paneles prefabricados de fachada.

Estos elementos están sometidos a los agentes exteriores en circunstancias desfavorables: agua, viento, cambios bruscos de temperatura, polución, etc.

Por eso, si se quiere garantizar una durabilidad adecuada, hay que cuidar al máximo la calidad del hormigón. Un hormigón de fachada, además de resistencia, debe tener una alta compacidad, es decir, tener una porosidad mínima.

\section{MATERIALES A EMPLEAR}

Además de las piedras y mármoles naturales de poca porosidad, se ha venido empleando la piedra artificial y el hormigón vibrado, hasta la aparición del procedimiento "SCHOKBETON", o sea, el del hormigón chocado; en el futuro, quizá el material más adecuado sea el hormigón polimerizado.

La llamada piedra artificial se consigue a base de un mortero - no de un hormigón- generalmente de cemento blanco y arenas procedentes de machaqueo de una piedra o mármol, cuyo color y aspecto se desea imitar. Este mortero se coloca en el molde -que la mayoría de las veces es de escayola- por simple vertido y sin vibrado, lo que requiere una relación agua-cemento muy alta, obteniéndose un producto muy poroso y retráctil. Esto tiene consecuencias funestas para la durabilidad de los elementos así fabricados: penetración de agua, heladicidad, corrosión de las armaduras, efectos expansivos del óxido, etc.; por otro lado, su débil resistencia no permite la prefabricación de paneles que durante su manejo, transporte o montaje están sometidos a fuertes tensiones.

La piedra artificial se queda reducida, pues, a aplacados y molduras de poco relieve; las cornisas y elementos de mayor vuelo dan serios disgustos con el transcurso del tiempo a viandantes, constructores y arquitectos; estos últimos responsables durante el período decenal que exige el actual Código Civil.

El hormigón vibrado se trata ya de un verdadero hormigón, sobre todo si se cuida la granulometría y la relación agua-cemento. Esta solución es muy adecuada para elementos estructurales y resistentes, sobre todo para piezas lineales, como pilares y vigas, o de gran volumen, ya que toda la masa se compacta por igual.

De todas formas, en el vibrado se produce el "efecto pared" como consecuencia del cual, los finos tienden a colocarse en la cara del molde y los áridos de mayor tamaño se retiran de la misma, por lo que se deben cuidar los recubrimientos. También se observa que las superficies en contacto con el molde quedan más porosas y con burbujas de aire.

Por último, el hormigón chocado que da nombre al sistema SCHOKBETON, parece ser el procedimiento ideal para elementos superficiales, tales como los paneles de fachada, las placas de gran área y los antepechos.

En el sistema SCHOKBETON el hormigón se vierte en pequeñas capas, colocando el molde sobre grandes mesas sacudidas que están sometidas a un movimiento vertical alternativo de gran amplitud - del orden de los $5 \mathrm{~mm}$ - y de pequeña frecuencia - unos 250 golpes por minuto-, es decir, lo contrario de la vibración de alta frecuencia que se emplea en los hormigones vibrados.

Gracias a este sistema, los áridos se van sedimentando por densidades, compactando la cara inferior que es la cara del molde, debido al efecto cinético de la caída; las gravillas o piedras mayores se van situando preferentemente en dicha cara - no hay "efecto pared"-, se cierra la granulometría y se compacta la superficie.

Además, debido a la proximidad de los gruesos a la cara, cuando se hace un tratamiento, por ligero que sea, de chorro de arena, lavado, etc., los áridos afloran inmediatamente dando un bello aspecto a la superficie manifestándose con intensidad el color de la piedra.

La figura 1 muestra un gráfico comparativo del hormigón chocado y el hormigón vibrado. En abscisas se muestra el tiempo en segundos y, por tanto, indica la frecuencia; y en ordenadas la amplitud. Aparte del efecto de sedimentación a que antes hemos aludido, se produce por la caida libre de la masa de hormigón fresco en el chocado una compactación sobre la cara inferior debido a 
Fig. 1

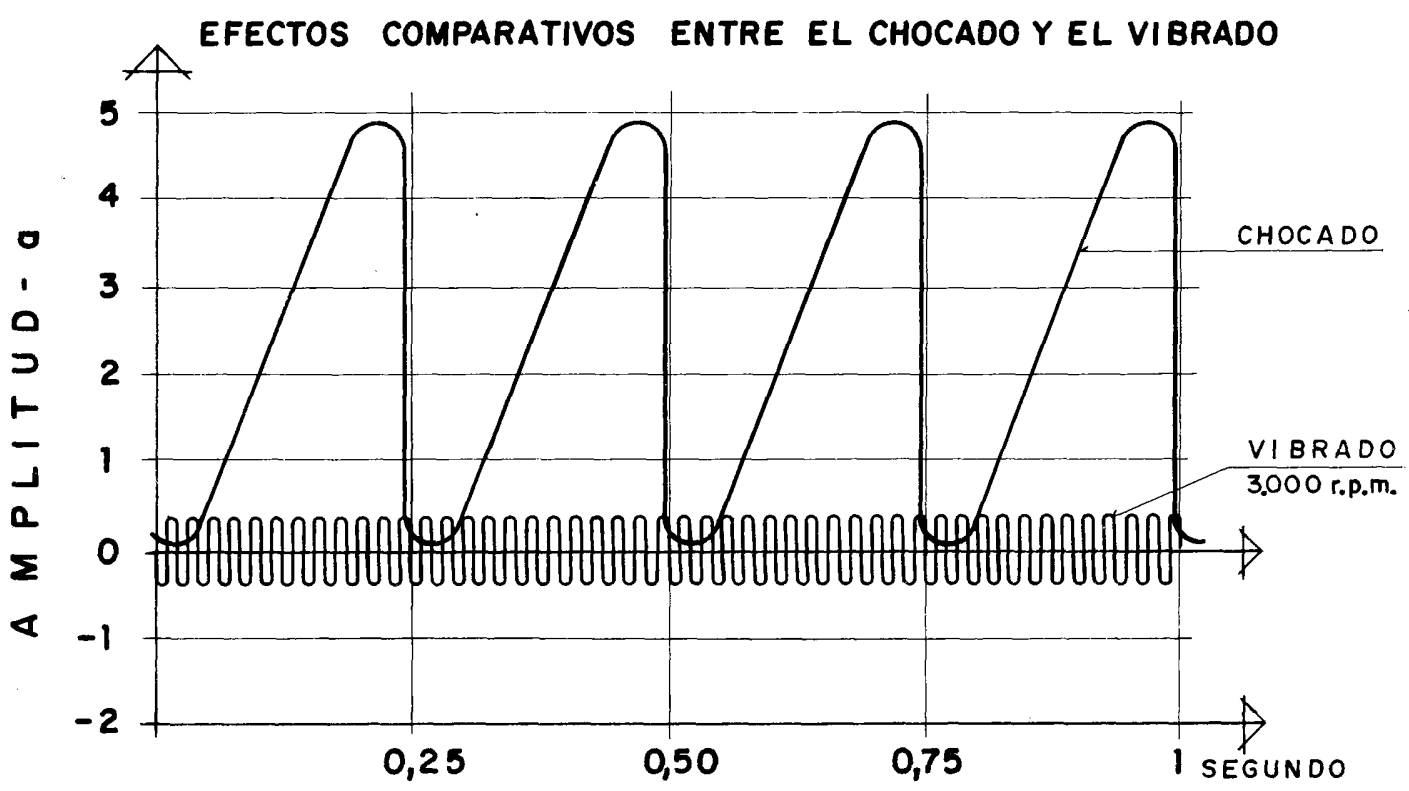

\begin{tabular}{|l|c|c|c|}
\cline { 2 - 4 } \multicolumn{1}{c|}{} & $\begin{array}{c}\text { FRE CUENCIA } \\
\text { r.P. } \mathbf{m}\end{array}$ & $\begin{array}{c}\text { A MPLITUD } \\
\mathbf{m} \mathbf{~ m}\end{array}$ & ACELERACION \\
\hline CHOCADO & 250 & 5 & $0.35 \mathrm{~g}$ \\
\hline VIBRADO & 3.000 & 0.4 & $4.00 \mathrm{~g}$ \\
\hline $\begin{array}{l}\text { VIBRA DO ALTTA } \\
\text { FRECUENCIA }\end{array}$ & 12.000 & 0.2 & $32.00 \mathrm{~g}$ \\
\hline
\end{tabular}

la fuerza viva que vale $\mathbf{0 . 5} \mathbf{m} \mathbf{~ v}^{2}$, en la que $\mathbf{m}$ es la masa que cae, del orden de 8 ó $10 \mathrm{~cm}$ de espesor, y $\mathbf{v}$ es la velocidad de caída que depende de la altura. Esta fuerza alcanza valores muy importantes que contribuyen a compactar la cara inferior que luego es la que quedará vista en la fachada.

La figura 2 muestra un cuadro comparativo de los valores de peso específico aparente, así como para la absorción superficial de agua y la capilaridad de los tres materiales que hemos analizado a las 24 horas.

Los resultados son tan elocuentes que no requieren hacer el menor comentario.

Por último diremos que, en el futuro, se utilizarán los hormigones polimerizados que puede ser la solución perfecta. En esencia consiste en impregnar el hormigón fabricado por los métodos usuales, con un monómero que luego se polimeriza in situ. De esta manera, los poros o canalitos que queden en el hormigón, por pequeños que sean, se rellenan por este monómero (que puede ser el metacrilato de metilo por su baja viscosidad) y que luego es polimerizado por efecto de vapor o temperatura. Se obtienen asi hormigones sin absorción y con resistencia que rebasan fácilmente los $1.000 \mathrm{~kg} / \mathrm{cm}^{2}$. Conseguido ya a nivel de laboratorio, la investigación busca actualmente las soluciones industriales. Todas las filiales de SCHOKBETON, en el mundo entero, estamos trabajando en este campo.

\section{METODO OPERATORIO}

Generalmente se cuenta con un laboratorio de hormigón y de Control de Calidad.

Una vez terminada la idea de color y textura por el arquitecto, se hacen unas placas de muestra para matizar estas caracteristicas.

Seleccionados los áridos se estudia la granulometría adecuada por el método Rothfuchs, ya que los métodos de dosificación usuales no son válidos en este caso, en que se llega a mezclar hasta seis áridos diferentes para un tipo de hormigón arquitectónico. No se utilizan colorantes químicos. 


\section{ENSAYOS COMPARATIVOS DE LABORATORIO}

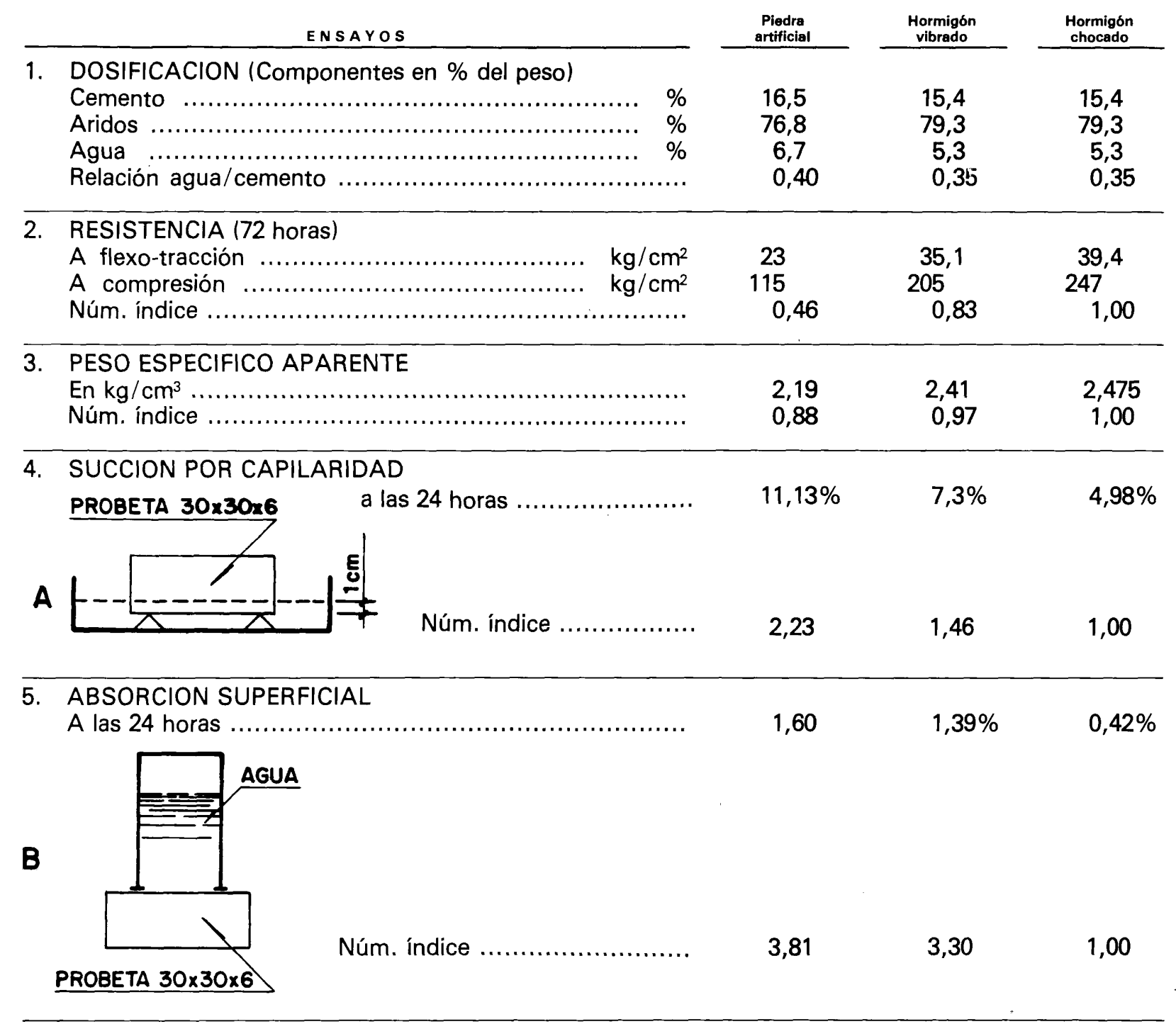

NOTAS: - Las probetas han sido tratadas al chorro de arena en la cara objeto del ensayo.

Fig. 2

- Todos los ensayos de absorción y succión se inician en las probetas desecadas en estufa a peso constante.

- El peso específico aparente se ha determinado después de desecadas las probetas a peso constante.

- La resistencia se ha determinado a las 72 horas de la fabricación.

Una vez determinada esta curva granulométrica, se hacen una serie de probetas de ensayo, para determinar la resistencia y, sobre todo, la absorción y la heladicidad, asi como la actitud al tratamiento final de terminación elegido por el arquitecto.

La fabricación está sometida a severo control de calidad. Los silos de áridos están automatizados, asi como las dos centrales de amasado a contracorriente, para conseguir una homogeneidad en la superficie vista; el transporte de hormigón a los moldes se hace por tolvas vibratorias para evitar la segregación, y el vertido del hormigón se hace en pequeñas capas de 3 a $5 \mathrm{~cm}$, actuando desde el primer vertido el chocado.

Las armaduras, las chapas de anclaje, los herrajes van electrosoldados con $\mathrm{CO}_{2}$ para evitar escoria que manche posteriormente por oxidación la superficie; la colocación de la armadura, los recubrimientos, las tolerancias dimensionales, etc., todo ello está sometido a un control estadistico de calidad, antes, durante y después del proceso.

\section{INDICACIONES PARA LA OBRA}

Una obra de prefabricado requiere una estructura con medidas bien determinadas, con tolerancias pequeñas, que permitan la correcta colocación de las piezas. Las tolerancias deseables son: 


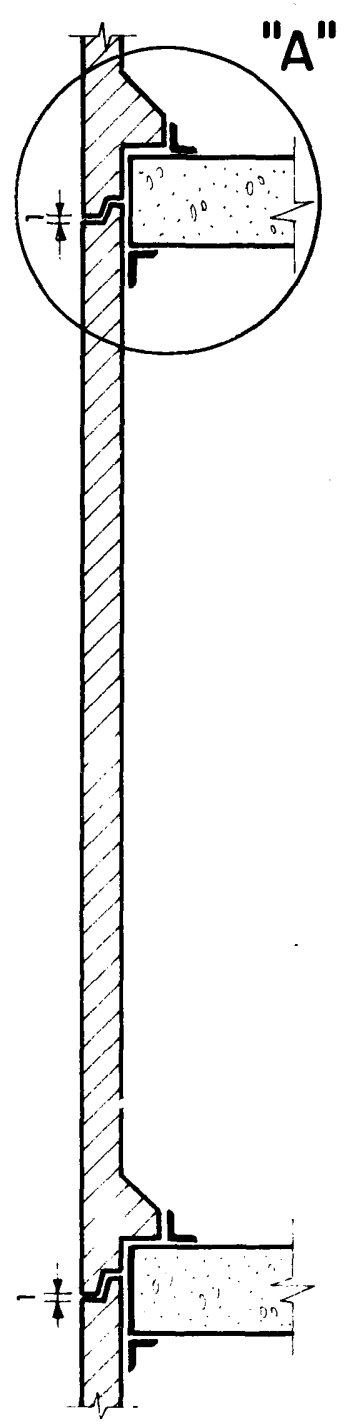

Fig. 3.1 SECCION PIEZAS GENERALES

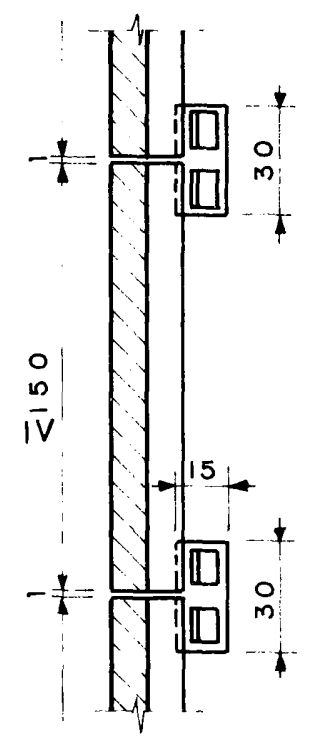

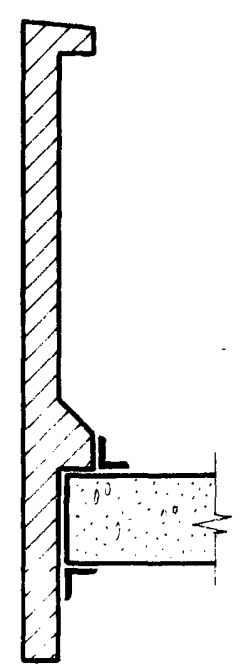

Fig. 3.4 SECCION ANTEPECHOS

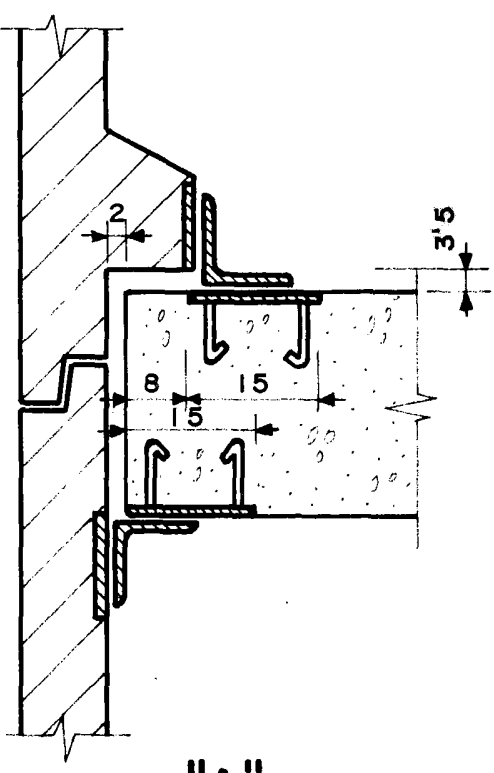

Fig. 3.2 DETALLE "A" DE ANCLAJES GENERALES

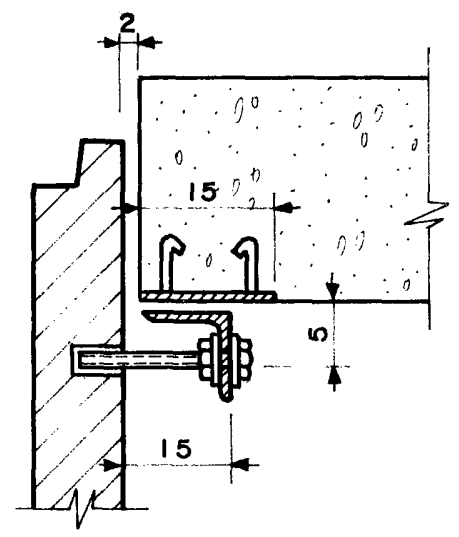

Fig. 3.3 DETALLE ESPECIAL PARA ANCLAJE ELASTICO

Fig. 3.5 PLANTA PIEZAS GENERALES 
Defectos de nivelación en bordes de forjados

$\pm 15 \mathrm{~mm}$

Espesor de los forjados

$+15 \mathrm{~mm}$

Plomos en los bordes de los forjados...

$\pm 15 \mathrm{~mm}$

Alturas de piso a piso

土 $10 \mathrm{~mm}$

Altura total de la parte afectada por el prefabricado.

土 $25 \mathrm{~mm}$

Para dejar las chapas empotradas y herrajes en las otras, lo mejor es consultar con la oficina técnica, correspondiente, quien una vez aceptada la obra dará planos detallados de las chapas a insertar en el hormigón de los forjados.

\section{TENGASE EN CUENTA QUE LAS CHAPAS NO PREVISTAS CREAN POSTERIORMENTE PROBLEMAS EN LA OBRA Y SOLUCIONES SIEMPRE CARAS E INCORRECTAS}

Para el caso de paneles en toda la altura de una planta, véase la fig. 3.1: la pieza apoya en el borde del forjado por medio del tacón de hormigón, y va soldado a la chapa de anclaje previamente empotrada en el hormigón. Esta chapa debe quedar a $8 \mathrm{~cm}$ del borde de la estructura (véase figura 3.2). En la parte superior también la pieza va soldada a una chapa de anclaje, prevista en la estructura, pero en este caso se coloca en el borde.

Cuando el panel se coloca en el borde de un voladizo flexible, en el que sean previsibles oscilaciones de la fachada, se debe colocar un anclaje libre, en la parte superior, como indica la figura 3.3.

En el caso de antepechos con cuelgue, la solución más adecuada es la fig 3.4 , es decir, soldado por arriba y por debajo del forjado. La colocación de los chapones de anclaje se hace igual que en el detalle de la fig. 3.2.

Para determinar en planta la situación de estos chapones de anclaje, debemos distinguir el caso de que los paneles o antepechos tengan una longitud, menor o mayor de 1,50 metros.

En el primer caso, se coloca la chapa de anclaje coincidiendo con la junta de separación de paneles y se aprovechan para fijar los dos paneles a la misma chapa; ésta suele tener, en estos casos, unas dimensiones de $300 \times 150 \times 10 \mathrm{~mm}$. En el segundo caso, es decir, con paneles de más de 1,50 m es preferible no soldar en las juntas, porque asi, se permite una mayor libertad de dilatación térmica de los paneles, y se suelen colocar chapas de anclaje de $150 \times 100 \times 10 \mathrm{~mm}$ (véase fig. 3.5).

No olvidar nunca que, en general, las chapas de anclaje, colocadas en la parte superior del forjado, van separadas del borde del forjado $8 \mathrm{~cm}$, para permitir el vuelo del tacón; en cambio, las que van colocadas en la parte inferior del forjado, se deben colocar al borde del mismo.

\section{CONSIDERACIONES GENERALES PARA EL DISEÑO DE PANELES}

Las fachadas prefabricadas de hormigón arquitectónico permiten la creación de nuevas formas y soluciones inviables con otras soluciones. Pero para que la solución no resulte encarecida, el arquitecto debe tener presente algunas recomendaciones o reglas:

\section{REGLA 1.a}

Al proyectar las piezas debe pensarse en el montaje. A veces, piezas que no tienen problemas de fabricación, a pesar de su tamaño o forma, crean grandísimos problemas de montaje. 
En general, el montaje con grúa-torre tiene limitaciones por el peso. La grúa-torre debe efectuar la descarga del camión $y$, a veces, esto exige una longitud de pluma excesiva. También los voladizos de las últimas plantas que sobresalen de la línea de fachada crean dificultades en el montaje. En la fotografia de la fig. 5.1 se observa que las grúas-torre se han colocado encima de la estructura de un gran edificio.

El montaje con auto-grúa permite mayor libertad de movimientos, pero a veces la altura excesiva del edificio imposibilita esta solución.

\section{REGLA 2. ${ }^{a}$}

Para obtener una economía importante es imprescindible una serie numerosa de piezas. Cada molde de prefabricado es muy caro y debe amortizarse en número suficientemente repetitivo de piezas, para no encarecerlas inútilmente.

Hay que estudiar con sumo cuidado la modulación de fachada, la situación de huecos, las soluciones de esquinas, etc., a fin de no encarecer el prefabricado.

También hay que pensar en el posible molde, de manera que sea factible su desmoldeo, sin dañar la pieza.

\section{REGLA 3.}

Para que la fachada permanezca limpia, conviene pensar en el desagüe de las piezas y cuidar los detalles de goterones, guardapolvos, etc., con mucho cuidado. Una superficie horizontal donde se deposita el polvo y la polución de la ciudad, producirá manchas y chorreones cuando aparezcan las primeras lluvias.

De todas formas, hay que huir de materiales porosos o poco compactos, como pueden ser la piedra artificial o el hormigón vibrado. El hormigón chocado es la solución más adecuada contra la contaminación y manchas de las fachadas.

Observando estas tres simples reglas se puede conseguir una fachada estética, racional, permanente y económica, y una obra que marque la personalidad de su autor.

\section{résumé}

\section{CARACTERISTIQUES DU BETON POUR LA PREFABRICATION DES FACADES}

\section{Antonio Puerta, \\ SCHOKBETON ESPAÑOLA, S. A.}

Dans cet article sont étudiés les avantages qu'ont le béton choqué du présent et le béton polymérisé du futur, sur les matériaux traditionnellement utilisés pour la préfabrication des façades: pierre et marbres naturels peu poreux, pierre artificielle et béton vibré.

En plus des caractéristiques de ces matériaux, sont données des indications pour l'exécution parfaite des travaux et certaines recommandations pour adopter la solution la plus esthétique, rationnelle, permanente et économique possible.

\section{summary}

CHARACTERISTICS OF CONCRETE USED IN PREFAB FACING MODULES

Antonio Puerta,

SCHOKBETON ESPANNOLA, S. A

This article studies the advantages offered now by shock concrete and those to be now by shock concrete and those to be over the traditional materials used in prefab facing modeles: various low porosity stones and marbles, artificial stone and vibrated concrete.

Besides describing the properties of such materials, the work gives some indications for their correct application in construction jobs and certain recommendations to make the adopted solutions as aesthetical, rational durable and economic as possible.

\section{zusammenfassung}

\section{MERKMALE DES BETONS FUER VORGEFERTIGTE FASSADEN}

Antonio Puerta

SCHOKBETON ESPAÑOLA, S. A.

In diesem Artikel werden die Vorteile untersucht, welche der gepralite Beton zur Zeit und der polymerisierte Beton in der Zukunft bei den üblicherweise zur Vorfertigung von Fassaden verwendeten Materielien wie: $\mathrm{Na}$ tursteine und -marmor geringer Porosität, Kunststeine und Rüttelbeton bieten können.

Neben der Beschreibung dieser Materialien werden Anweisungen und Hinwiese für die einwandfreie Durchführung dieser Bauten sowie einige Empfehlungen gegeben, damit die angenommene Lösung möglichst ästhetische, rationell, dauerhaft und wirtschaftlich ausfällt. 